\title{
Identification of interleukin-6 (IL-6) and squamous cell carcinoma (SCC) as amniotic fluid-specific markers*
}

\author{
Katsuhiko Naruse, Taketoshi Noguchi, Shozo Yoshida, Taihei Tsunemi, Hiroshi Shigetomi, \\ Hidekazu Oi, Hiroshi Kobayashi
}

Department of Obstetrics and Gynecology, Nara Medical University, Nara, Japan

Email: hirokoba@naramed-u.ac.jp

Received 24 April 2012; revised 21 May 2012; accepted 30 May 2012

\begin{abstract}
Objective: To determine if an amniotic fluid (AF)specific marker is present and if its concentration changes with the presence of labor. Study Design: Twenty-six healthy women who gave birth to healthy newborns at term during the period from July 2009 to January 2010 were included in the study. Six candidate markers were assessed by commercially available ELISA kits: interleukin (IL)-6, squamous cell carcinoma (SCC) antigen, insulin-like growth factor (IGFBP)-1, osteopontin (OPN), CA125, and sialyl Tn (STN). Results: The AF/maternal serum (MS) measurement based on IL-6 or SCC has proved to be superior to IGFBP-1, CA125, OPN and STN. Women with spontaneous labor at term had significantly higher IL-6 and IGFBP-1 concentrations in AF compared with those without labor. No significant differences were observed in the AF concentrations of SCC, OPN, CA125 and STN between women with labor and those not in labor. Conclusion: Our observation of IL-6 and SCC in AF may open a new area of research to assess their usefulness as biological markers of obstetrical disorders.
\end{abstract}

Keywords: Amniotic Fluid; Biomarker; Interleukin-6; Squamous Cell Carcinoma Antigen

\section{INTRODUCTION}

Several investigators have evaluated the diagnostic value of amniotic fluid (AF)-specific polypeptides or metabolites as indicators of obstetrical disorders, including premature rupture of membranes (PROM) [1-4] or amniotic fluid embolism (AFE) [5-10]. We have conducted a document retrieval to identify gene products that are spe-

\footnotetext{
"Conflict of interest: department of obstetrics and gynecology, Nara Medical University, the chairman and professor, Hiroshi Kobayashi, had received subscription from Alfresa Pharma Corporation in the past one year. The other authors declare that they do not have any conflict of interest.
}

cifically present only in $\mathrm{AF}$ but not present in the maternal serum (MS) or proteins/polypeptides that are present in AF at concentrations extremely higher than those in the MS. Proteomic technologies have been predominantly used in the research to discover new AFspecific markers [11-15]. We have previously reported many candidate markers [16]. Enriched protein functions were tumor markers, cell proliferation and embryonic development, metabolism, nervous system, cytokines, immune or complement processes, signaling, cell adhesion and motility, hormones, detoxification system, and metal carrier [16].

In the present study, among these candidate markers, the following six antigens, interleukin (IL)-6, insulin-like growth factor-binding protein (IGFBP)-1, osteopontin (OPN), Squamous cell carcinoma (SCC) antigen, CA125 and sialosyl Tn (STN), were selected, since the specific ELISA has been commercially available. We have examined if these proposed markers are over expressed specifically in AF and if their concentration changes with the presence of labor.

\section{MATERIALS AND METHODS}

\subsection{Study Population and AF Collection}

Twenty-six healthy women who gave birth to healthy newborns at term during the period from July 2009 to January 2010 at the Nara Medical University Hospital, Nara, Japan, were included in the study. Pregnant women who presented to our hospital were asked to participate. Two groups of women were recruited: 1) 7 term pregnant women (gestational age, 37 - $41 \mathrm{wk}$ ) carrying a single fetus and undergoing elective cesarean section before labor, whose peripheral bloods were taken just prior to operation (term maternal blood); 2) 9 term pregnant women undergoing elective cesarean section before labor, whose amniotic fluids were taken (AF without labor); 3) 10 similar term pregnant women undergoing emergency cesarean section after spontaneous labor (all women were in the active phase of labor of less than 5 minutes 
interval), whose amniotic fluids were taken (AF with labor). The main indications for urgent and emergency cesareans were prolonged labor and presumed fetal compromise. Subjects with any symptom of infection were excluded from the study. No subject had a comorbid condition such as hypertension, diabetes, asthma, congenital heart disease, kidney disease, connective tissue disorders, and autoimmune disease. All neonates had normal anatomies. This study was approved by the Nara Medical University ethics committee. Informed consent was obtained from all mothers. Clinical data were obtained by two investigators (K.N. and T.N.) and recorded using a standardized data sheet. The women's ages, gestational ages, and parity did not differ significantly between the three groups.

\subsection{Sampling}

Maternal blood was obtained from a cannulated vein a few minutes prior to surgery. AF was obtained during surgery by one investigator (K.N.) and immediately filtered through $0.45 \mu \mathrm{m}$ pore filter (Millex, Millipore Co. Ltd., Billerica, MA) to avoid the debris. After centrifugation at $4^{\circ} \mathrm{C}$, MS and $\mathrm{AF}$ were immediately frozen and stored at $-80^{\circ} \mathrm{C}$ until further analysis.

\subsection{Measurements of Six Biomarker Levels in Maternal Serum and Amniotic Fluid}

These antigens were assessed by commercially available ELISA kits: IL-6, R\&D systems, Inc. (Minneapolis, MN); Squamous cell carcinoma (SCC) antigen, Osteopontin, STN, and CA125, ECLIA kits, BML Co.Ltd. (Tokyo, Japan); and IGFBP-1, Human ELISA kit, Phoenix Pharmaceuticals, Inc. (Burlingame, CA). Every sample was run in duplicate. Differences between the two measurements were minimal (intra-assay precision coefficients of variation equals $5 \%-8 \%$ ). Statistical analysis was performed using the Mann-Whitney U test (SPSS 15.0J, SPSS Japan Inc., Japan). Statistical significance was set at $p<0.05$. All values are expressed as the mean \pm S.E.M.

\section{RESULTS}

Figure 1 is a scatter diagram with bar graphs for $\mathrm{AF}$ values of IL-6, IGFBP-1, SCC, osteopontin, CA125, and STN. With the use of a commercially available immunoassay kit developed to detect these antigens, elevated concentrations of the markers are frequently found in AF: IL-6 $(1031.90 \pm 499.14 \mathrm{pg} / \mathrm{ml}$ for without labor and $7347.94 \pm 1600.22 \mathrm{pg} / \mathrm{ml}$ for with labor, $\mathrm{p}<0.05$ ),
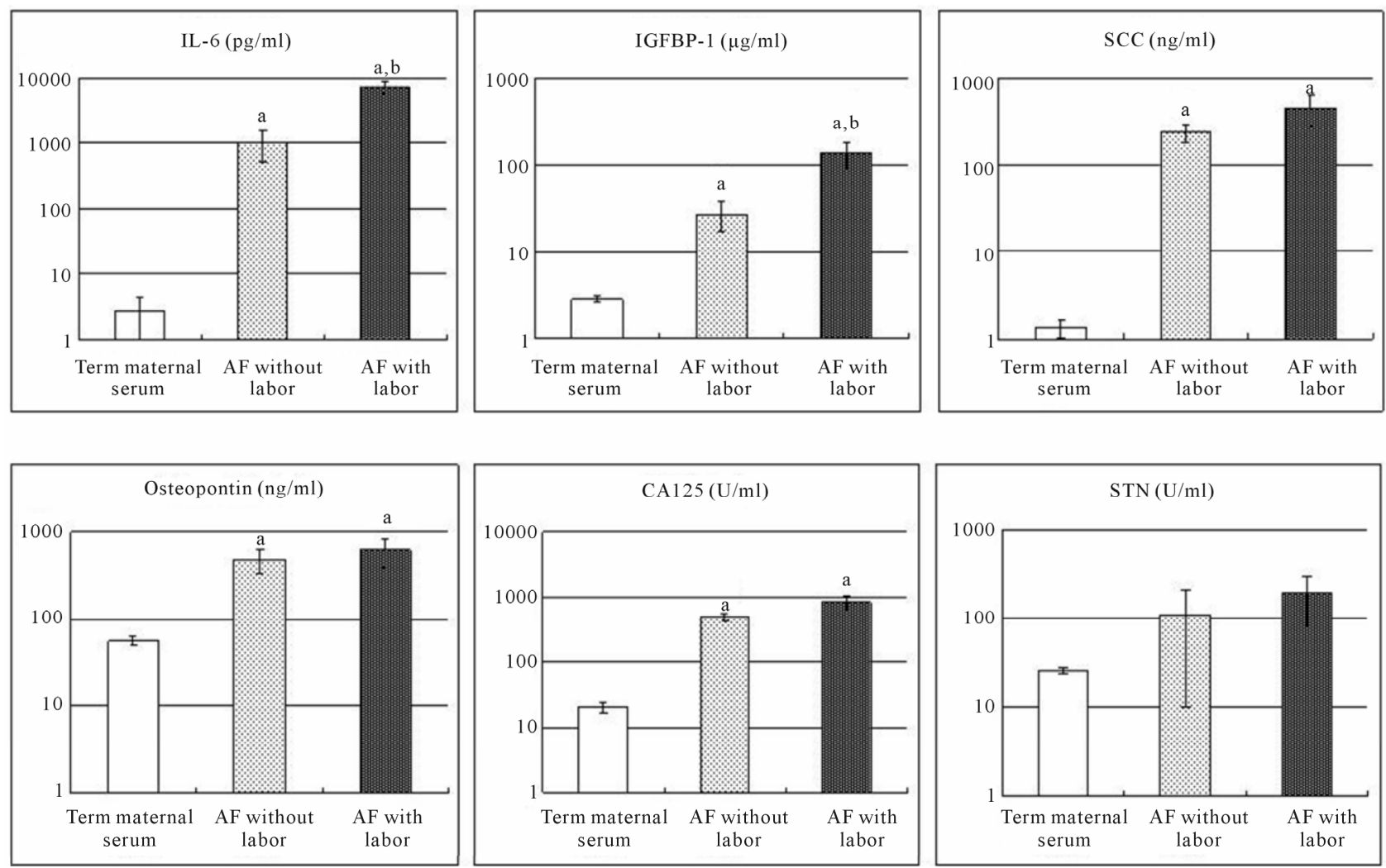

Figure 1. A scatter diagram with bar graphs of MS and AF concentrations of IL-6, IGFBP-1, osteopontin, SCC, STN, and CA125 in women with elective cesarean section before labor and emergency cesarean section after labor. Data are present as mean \pm S.E.M. a: $\mathrm{p}<0.05$ vs term maternal serum; $\mathrm{b}: \mathrm{p}<0.05$ vs AF without labor. 
IGFBP-1 $(27.09 \pm 9.86 \mu \mathrm{g} / \mathrm{ml}$ for without labor and $135.67 \pm 44.45 \mu \mathrm{g} / \mathrm{ml}$ for with labor, $\mathrm{p}<0.05)$, SCC $(240.08 \pm 57.03 \mathrm{ng} / \mathrm{ml}$ for without labor and $451.58 \pm$ $178.15 \mathrm{ng} / \mathrm{ml}$ for with labor), osteopontin (480.32 \pm $144.87 \mathrm{ng} / \mathrm{ml}$ for without labor and $611.54 \pm 217.00$ $\mathrm{ng} / \mathrm{ml}$ for with labor), CA125 (491.12 $\pm 62.21 \mathrm{U} / \mathrm{ml}$ for without labor and $840.70 \pm 186.64 \mathrm{U} / \mathrm{ml}$ for with labor), and STN $(108.63 \pm 98.57 \mathrm{U} / \mathrm{ml}$ for without labor and $191.05 \pm 107.76 \mathrm{U} / \mathrm{ml}$ for with labor).

Serum level of each marker was not markedly elevated and below the cut-off value. Very high AF/MS ratio was found in IL-6 (AF/MS = 395 for without labor and 2811 for with labor), followed by SCC (AF/MS = 179 for without labor and 336 for with labor), IGFBP-1 (AF/MS = 9 for without labor and 47 for with labor), CA125 $(\mathrm{AF} / \mathrm{MS}=24$ for without labor and 41 for with labor), osteopontin (AF/MS $=9$ for without labor and 11 for with labor), and STN (AF/MS $=4$ for without labor and 8 for with labor). The AF/MS measurement based on IL-6 or SCC has proved to be superior to IGFBP-1, CA125, osteopontin, and STN. Patients after labor showed significantly higher IL-6 and IGFBP-1 concentrations in AF compared with women before labor. Comparison of AF SCC levels between before and after labor was not significant. Osteopontin, STN and CA125 in AF and MS did not also change significantly with or without labor.

\section{DISCUSSION}

Gene expression profiling and proteomics analyses were used to analyze biological samples to identify the AFspecific genes and their products. Many candidates have been discovered and reported as shown in reference [16]. In the present study, AF and MS were collected at term, either before (elective caesarean section) or after the onset of labor (emergency caesarean section). We found that IL- 6 and SCC are present in AF at concentrations extremely higher than those in the MS $(11,14)$. It appears likely that both are AF-specific proteins.

The AF IL-6 levels increase in late gestation. A role in regulation of labor onset is suggested by observations that IL-6 up-regulates expression of genes controlling prostaglandin synthesis and signaling [17]. IL-6 might be produced by macrophages, mast cells, trophoblasts, decidua cells and amniotic fluid cells [18]. There is strong evidence that IL-6 in AF is a clinically useful test to predict preterm birth [19]. IL-6 might increase with both types of labor, preterm and term, with a greater change at the onset of labor.

On the other hand, the AF SCC levels were significantly higher and did not change with the onset of labor. In uncomplicated pregnancies, MS SCC levels at term gestation were similar to nonpregnant levels. To date, the exact source and biological role of elevated antigens are not clearly defined. Further work is necessary to evaluate the biological function of SCC for preterm and term labor.

Among the marker used in clinical practice, several investigators have evaluates the diagnostic value of IGFBP-1 as an indicator of PROM. However, contamination of the cervicovaginal samples by maternal blood can induce false positive results. The IGFBP-1 levels in MS at term are $>1000 \mathrm{ng} / \mathrm{ml}$, whereas the SCC concentrations are $<5 \mathrm{ng} / \mathrm{ml}$. The SCC test might be unaffected by contamination. SCC may have high predictive values for diagnosing PROM.

In addition, since AFE is a dramatical complication in pregnancy that occurs when AF enter the maternal circulation, the method for detecting the AF-specific antigens such as IL-6 and SCC in the MS may be a novel method for diagnosis of AFE. If AFE was verified by medicolegal autopsy, immunostaining for IL-6 or SCC in the affected lung may be a sensitive method to confirm AFE patients because these antibodies are commercially available. These markers would become the favorable biomarkers for detecting AFE. The test based on IL-6 or SCC seems to be a more sensitive bedside test compared with the conventional tests based on STN for the detection of entry of AF into maternal circulation $(9,10)$.

A limitation of this study should be noted. The sample size and small number may have limited our ability to detect small differences. So far, we have never determined the cervicovaginal fluid or MS levels in patients with PROM or AFE, respectively. Notwithstanding these limitations, our observation of SCC and IL-6 in AF opens a new area of research to assess their usefulness as biological markers of PROM or AFE. The future clinical study will evaluate whether or not each proposed marker is ready for clinical applications. Larger and validation studies are also needed to determine the true value of these new biomarkers.

\section{ACKNOWLEDGEMENTS}

Supported by a grant (Grant-in-aid for Scientific Research (H. Kobayashi, H. Oi and K. Naruse)) from the Ministry of Education, Science, and Culture of Japan and by a grant from Alfresa Pharma Corporation (2-2-9 Kokumachi, Chuo-ku, Osaka 540-8575, Japan (H. Kobayashi and K. Naruse)).

\section{REFERENCES}

[1] Rutanen, E.M., Pekonen, F. and Kärkkäinen, T. (1993) Measurement of insulin-like growth factor binding protein-1 in cervical/vaginal secretions: Comparison with the ROM-check membrane immunoassay in the diagnosis of ruptured fetal membranes. Clinica Chimica Acta, 214, 73-81. doi:10.1016/0009-8981(93)90304-M

[2] Medina, T.M. and Hill, D.A. (2006) Preterm premature 
rupture of membranes: Diagnosis and management. American Family Physician, 73, 659-664.

[3] Albayrak, M., Ozdemir, I., Koc, O., Ankarali, H. and Ozen, O. (2011) Comparison of the diagnostic efficacy of the two rapid bedside immunoassays and combined clinical conventional diagnosis in prelabour rupture of membranes. European Journal of Obstetrics \& Gynecology Reproductive Biology, 158, 179-182. doi:10.1016/j.ejogrb.2011.04.041

[4] van der Ham, D.P., van Melick, M.J., Smits, L., Nijhuis, J.G., Weiner, C.P., van Beek, J.H., Mol, B.W. and Willekes, C. (2011) Methods for the diagnosis of rupture of the fetal membranes in equivocal cases: A systematic review. European Journal of Obstetrics \& Gynecology Reproductive Biology, 157, 123-127. doi:10.1016/j.ejogrb.2011.03.006

[5] Conde-Agudelo, A. and Romero, R. (2009) Amniotic fluid embolism: An evidence-based review. American Journal of Obstetrics \& Gynecology, 201, 445.e1-e13. doi:10.1016/j.ajog.2009.04.052

[6] Kanayama, N., Yamazaki, T., Naruse, H., Sumimoto, K., Horiuchi, K. and Terao, T. (1992) Determining zinc coproporphyrin in maternal plasma-A new method for diagnosing amniotic fluid embolism. Clinical Chemistry, 38, 526-529.

[7] Kobayashi, H., Ohi, H. and Terao, T. (1993) A simple, noninvasive, sensitive method for diagnosis of amniotic fluid embolism by monoclonal antibody TKH-2 that recognizes NeuAc alpha 2-6GalNAc. American Journal of Obstetrics \& Gynecology, 168, 848-853.

[8] Kobayashi, H., Ooi, H., Hayakawa, H., Arai, T., Matsuda, Y., Gotoh, K. and Tarao, T. (1997) Histological diagnosis of amniotic fluid embolism by monoclonal antibody TKH-2 that recognizes NeuAc alpha 2-6GalNAc epitope. Human Pathology, 28, 428-433.

[9] Oi, H., Kobayashi, H., Hirashima, Y., Yamazaki, T., Kobayashi, T. and Terao, T. (1998) Serological and immunohistochemical diagnosis of amniotic fluid embolism. Seminars in Thrombosis and Hemostasis, 24, 479-484. doi:10.1016/S0046-8177(97)90031-9

[10] Oi, H., Naruse, K., Noguchi, T., Sado, T., Kimura, S., Kanayama, N., Terao, T. and Kobayashi, H. (2010) Fatal factors of clinical manifestations and laboratory testing in patients with amniotic fluid embolism. Gynecologic and Obstetric Investigation, 70, 138-144. doi: $10.1159 / 000313302$
[11] Suminami, Y., Nawata, S. and Kato, H. (1998) Biological role of SCC antigen. Tumour Biology, 19, 488-493. doi: $10.1159 / 000030042$

[12] Takeshima, N., Suminami, Y., Takeda, O., Abe, H. and Kato, H. (1993) Origin of CA125 and SCC antigen in human amniotic fluid. Asia Oceania Journal of Obstetrics and Gynaecology, 19, 199-204. doi:10.1111/j.1447-0756.1993.tb00373.x

[13] Khosravi, J., Krishna, R.G., Bodani, U., Diamandi, A., Khaja, N., Kalra, B. and Kumar, A. (2007) Immunoassay of serine-phosphorylated isoform of insulin-like growth factor (IGF) binding protein (IGFBP)-1. Clinical Biochemistry, 40, 86-93. doi:10.1016/j.clinbiochem.2006.07.004

[14] Menon, R., Camargo, M.C., Thorsen, P., Lombardi, S.J. and Fortunato, S.J. (2008) Amniotic fluid interleukin-6 increase is an indicator of spontaneous preterm birth in white but not black Americans. American Journal of Obstetrics and Gynecology, 198, 77.e1-e7. doi:10.1016/j.ajog.2007.06.071

[15] Qu, X., Yang, M., Zhang, W., Liang, L., Yang, Y., Zhang, Y., Deng, B., Gao, W., Liu, J., Yang, Q., Kong, B. and Gong, F. (2008) Osteopontin expression in human decidua is associated with decidual natural killer cells recruitment and regulated by progesterone. In Vivo, 22, 55-61.

[16] Kobayashi, H., Naruse, K., Sado, T., Noguchi, T., Yoshida, S., Shigetomi, H., Onogi, A. and Oi, H. (2011) Search for amniotic fluid-specific markers: Novel biomarker candidates for amniotic fluid embolism. The Open Women's Health Journal, 5, 7-15. doi:10.2174/1874291201105010007

[17] Robertson, S.A., Christiaens, I., Dorian, C.L., Zaragoza, D.B., Care, A.S., Banks, A.M. and Olson, D.M. (2010) Interleukin-6 is an essential determinant of on-time parturition in the mouse. Endocrinology, 151, 3996-4006. doi:10.1210/en.2010-0063

[18] Zenclussen, A.C., Blois, S., Stumpo, R., Olmos, S., Arias, K., Borel, I.M., Roux, M.E. and Margni, R.A. (2003) Murine abortion is associated with enhanced interleukin6 levels at the feto-maternal interface. Cytokine, 24, 150160. doi:10.1016/j.cyto.2003.08.002

[19] Krupa, F.G., Faltin, D., Cecatti, J.G., Surita, F.G. and Souza, J.P. (2006) Predictors of preterm birth. International Journal of Gynaecology \& Obstetrics, 94, 5-11. doi:10.1016/j.ijgo.2006.03.022 